\title{
Dissolution Velocity of Zinc Ferrite in Acids
}

\author{
Wlodzimierz Wolski* \\ Irena Okonska Koztowska**
}

\begin{abstract}
Wlodzimierz Wolski and Irena Okonska Kozlowska: Dissolution Velocity of Zinc Ferrite in Acids

The kinetics of the system $\mathrm{ZnFe}_{2} \mathrm{O}_{4} / \mathrm{H}^{+}$has been examined in various concentrations of hydrochloric and nitric acids separately and in their mixtures. It was found that in nitric acid independent of its concentration the process is non-stoichiometric one, giving after the same lapse of time more zinc cations to the solution than the stoichiometricity requires. This behaviour can be observed in both dilute and concentrated $\mathrm{HNO}_{3}$.
\end{abstract}

In the presence of hydrochloric acid the same ferrite displays quite different way of dissolution; here according to the analytical data, the amounts of ferric and zinc ions do indicate the molar solubility. Rather surprisingly, however, the best conditions in order to dissolve stoichiometrically this compound in acids under investigation, have been achieved for the mixture of $2 \mathrm{~N} \mathrm{HNO}_{3}$ and $2 \mathrm{~N} \mathrm{HCl}$ even, when the proportion of $\mathrm{HCl}: \mathrm{HNO}_{3}$ had been as small as $1: 20$.

The results of temperature effects examination $\left|20^{\circ}, 40^{\circ}, 60^{\circ} \mathrm{C}\right|$ upon this reaction showed, as to be expected, much more greater rate of dissolution in higher temperatures. The findings allowed to establish the kinetic equation and calculate the rate constants $\mathrm{K}$ and $\mathrm{k}$. The most accurately they fit when put in the following equation: $\frac{\mathrm{dx}}{\mathrm{dt}}=\mathrm{s}_{0} \mathrm{k}(1-\mathrm{x})^{3} ; \mathrm{K}=\frac{1}{\mathrm{t}}\left[\frac{1}{(1-\mathrm{x})^{2}}-1\right] ; \mathrm{K}=2 \mathrm{ks}_{0}$, where $\mathrm{s}_{0}$ is the specific area.

(Received December 29, 1970)

\section{Introduction}

The history of studies concerning the dissolution kinetics of ferrites is not long and in the world literature there is not much information on the velocity of passing into solution of the compounds of this class.

Only few authors investigated the solubility of zinc ferrite $|1,2,3,4|$. They tried to go further into the dissolution conditions of zinc ferrite in sulphuric acid and to establish the kinetics of this process on the ground of dissolution velocity, when taking into consideration such variables as : surface area, method of ferrite synthesis and heat treatment of raw materials and prepared ferrites. They arrived at the conclusion that the kinetics of this process is best represented by the equation:

$$
\frac{\mathrm{dx}}{\mathrm{dt}}=\mathrm{KS}_{0}|1-\mathrm{x}|^{\frac{2}{3}}
$$

and after integrating

$$
\begin{gathered}
1-|1-\mathrm{x}|^{\frac{1}{3}}=\mathrm{K} \mathbf{t} \\
\mathrm{K}=\frac{1}{3} \mathbf{k ~ S}_{0}
\end{gathered}
$$

where:

* Magnetochemistry Department of Adam Mickiewicz University Poznan, Poland

** General Physics Institute of Siletion University, Katowice, Poland 
$\mathrm{x}$-solubility $|\%|$

t-time in $\min$.

K-apparent constant velocity depending on surface area $\left|\min ^{-1}\right|$.

$\mathrm{k}$-constant velocity independent of surface area $\left|\mathrm{g} \cdot \mathrm{min}^{-1} \cdot \mathrm{m}^{-2}\right|$

$\mathrm{S}_{0}$-initial surface area $\left|\mathrm{m}^{2} \cdot \mathrm{g}^{-1}\right|$.

In the work now to be described the results of investigations concerning the solubilities of zinc ferrite in the nitric and hydrochloric acids are presented together with the kinetics of this process, which seems to proceed according to a different equation of the constant of dissolution velocity.

\section{Experimental}

The zinc ferrite under investigation was synthesized by ceramic method, i.c. by calcinating at $1200^{\circ} \mathrm{C}$ the previously mixed and pressed zinc and iron oxides of analytical purity. The correctness of synthesis was checked by the Debye-Scherrer X-ray identification method which recognized the presence of only pure zinc ferrite phase. Before starting the experiments, the ferrite was powdered and then screened to obtain a uniform and known grain fraction. The surface area of the ferrites was determined by low-temperature krypton sorption method. This surface area was $0.4 \mathrm{~m}^{2} \cdot \mathrm{g}^{-1}$.

The ferrite dissolution was carried out in a three-necked vessel equipped with thermometer and stirrer. $500 \mathrm{ml}$ of acid $15 \mathrm{~N} \mathrm{HNO}_{3}, 1 \mathrm{ON} \mathrm{HNO}, 12 \mathrm{~N} \mathrm{HNO}_{3}, 5 \mathrm{~N} \mathrm{HCI}, 8 \mathrm{~N} \mathrm{HCI}, 10 \mathrm{~N}$ $\mathrm{HCl}$ and their mixes/ were introduced into the vessel heated in a ultrathermostat to the measurement temperature. Then followed starting of stirrer and introduction of an accurate weight of ferrite. A stop-watch was switched on while pouring the weighed portion. $1 \mathrm{ml}$ of the solution was taken out for analysis at definite intervals. Zinc and iron ions contained in the solution were determined by analytical way.

In the presence of iron, the ions of zinc were determined by the direct titrimetric method with EDTA using eriochrome black $\mathrm{T}$ as indicator. Triethanolamine was used to eliminate the influence of iron upon the results of zinc titration. Preliminary precipitation of iron with ammonia or pyridine was carried out to make sure whether the masking with triethanolamine does not cause any error in the zinc content determination. After filtration of ferric hydroxide, the filtrate was titrated with $0.01 \mathrm{M}$ solution of EDTA. Triethanolamine was found to have no effect upon zinc determination.

Iron was determined in the presence of sulphosalicylic acid, which forms only one kind of complex compound with ferric ions in acid medium $\mid \mathrm{pH}$ maks. 3|. This method is suitable for iron determination in acid medium and in the presence of other ions. The resulting red violet solution was titrated with $0.01 \mathrm{M}$ EDTA solution until colour vanishing. As EDTAferric iron complex is stronger than sulphosalicylic acid-ferric iron complex the red colour disappears in the end point of titration.

The results of titrations were checked polarographically using ammonia puffer with triethanolamine as supporting electrolyte. Maxima were suppressed with $1 \%$ polyacrylo-, amide solution. Under these conditions distinct waves are obtained both for zinc and iron ions. Standard curves method was applied. Polarographic results were very close to the titrimetric ones. 


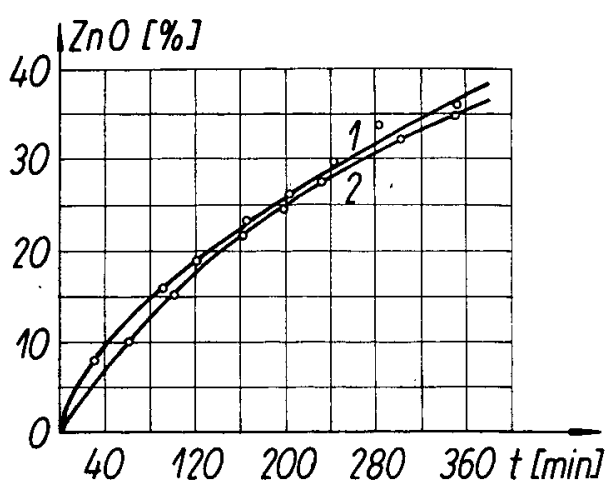

Fig. $1 \mathrm{Zn}^{2+}$ determination by 1 /polarographie method 2 /titration.

Fig. 1 gives an example of the usefulness of both methods in the case of zinc determination. The results relate to zinc ferrite dissolution in $8.5 \mathrm{~N} \mathrm{HCl}$ at $40^{\circ} \mathrm{C}$.

\section{Results}

First of all the analytical determinations of zinc and iron ions' concentration in the solution proved that zinc ferrite does not dissolve stoichiometrically neither in nitric nor in hydrochloric acid at low concentrations as well as in some of their mixes. This effect was already observed while dissolving other ferrites $|5|$ as well as zinc ferrite in the diluted nitric acid and it made to carry out quite a number of experiments in order to select conditions permitting to

Table 1 Solubility of zinc ferrite $\mathrm{ZnFe}_{2} \mathrm{O}_{4}$ versus nitric acid concentration.

\begin{tabular}{|c|c|c|c|c|c|c|}
\hline $\begin{array}{l}\text { Item } \\
\text { No }\end{array}$ & $\begin{array}{c}\text { Acid } \\
\text { concentration, } \\
\text { temperature }\end{array}$ & $\begin{array}{c}\text { Dissol. } \\
\text { time }\end{array}$ & $\begin{array}{c}\mathrm{ZnO} \\
\text { solubility }\end{array}$ & $\begin{array}{c}\mathrm{Fe}_{2} \mathrm{O}_{3} \\
\text { solubility }\end{array}$ & $\underset{\text { solubility }}{\mathrm{ZnO}}$ & $\begin{array}{c}\mathrm{Fe}_{2} \mathrm{O}_{3} \\
\text { solubility }\end{array}$ \\
\hline & normality & $\min$. & $\frac{\mathrm{mg}}{100 \mathrm{ml} \mathrm{of} \mathrm{sol} .}$ & $\frac{\mathrm{mg}}{100 \mathrm{ml} \text { of sol. }}$ & $\%$ & $\%$ \\
\hline 1 & 2 & 3 & 4 & 5 & 6 & 7 \\
\hline \multirow{6}{*}{1.} & \multirow{6}{*}{$5 \mathrm{~N} \mathrm{HNO}_{3} ; 40^{\circ} \mathrm{C}$} & 20 & 3.37 & 3.31 & 1.0 & 0.5 \\
\hline & & 40. & 5.06 & 4.63 & 1.5 & 0.7 \\
\hline & & 60 & 6.75 & 4.63 & 2.0 & 0.7 \\
\hline & & 80 & 8.44 & 6.62 & 2.5 & 1.0 \\
\hline & & 180 & 10.80 & 13.24 & 3.2 & 2.0 \\
\hline & & 300 & 13.50 & 16.56 & 4.0 & 2.5 \\
\hline \multirow{6}{*}{2.} & \multirow{6}{*}{$10 \mathrm{~N} \mathrm{HNO}_{3}, 40^{\circ} \mathrm{C}$} & 20 & 4.05 & 3.32 & 1.2 & 0.5 \\
\hline & & 40 & 6. 75 & 5.29 & 2.0 & 0.8 \\
\hline & & 60 & 8.44 & 7.94 & 2.5 & 1.2 \\
\hline & & 80 & 10.12 & 9.93 & 3.0 & 1.5 \\
\hline & & 180 & . $\quad 15.19$ & 17.88 & 4.5 & 2.7 \\
\hline & & 300 & 18.56 & 21.19 & 5.5 & 3.2 \\
\hline \multirow{6}{*}{3.} & \multirow{6}{*}{$12 \mathrm{~N} \mathrm{HNO}_{3}, 40^{\circ} \mathrm{C}$} & 20 & 5.06 & 5.29 & 1.5 & 0.8 \\
\hline & & 40 & 6.75 & 8.61 & 2.0 & 1.3 \\
\hline & & 60 & 10.12 & 12.52 & 3.0 & 1.9 \\
\hline & & 80 & 10.12 & 13.24 & 3.0 & 2.0 \\
\hline & & 180 & 15.19 & 23.18 & 4.5 & 3.5 \\
\hline & & 300 & 20.25 & 27.82 & 6.0 & 4.2 \\
\hline
\end{tabular}


obtain a real molar solubility, that is to say such conditions when one atom of zinc and two atoms of iren go into solution at a time. By way of example in Table 1 is set out the behaviour of zinc ferrite in the presence of 5,10 and $12 \mathrm{~N}$ nitric acid at $40^{\circ} \mathrm{C}$. As seen from this Table under these conditions far more zinc than iron go into solution.

Table 2 represents the behaviour of zinc ferrite in 5,8 , and $10 \mathrm{~N}$ hydrochloric acid at $40^{\circ} \mathrm{C}$. Except the first minutes, the molar dissolution takes place i.e. the same amount of $\mathrm{ZnO}$ and $\mathrm{Fe}_{2} \mathrm{O}_{3}$ pass into solution at a time. $\mathrm{ZnO}$ prevails in the case of $5 \mathrm{~N} \mathrm{HCl}$, its preponderance almost disappears in $8 \mathrm{~N} \mathrm{HCl}$, whereas at high concentration of $\mathrm{HGl} / 10 \mathrm{~N} /$ ferric oxide prevails in solution during initial period of dissolution.

Kinetics of molar dissolution in hydrochloric acid being more promising for investigations, one attempted to get more knowledge about the effect of temperature upon this process, using hydrochloric acid of always the same normality $\left[8 \mathrm{~N} \mid\right.$ at 20,40 and $60^{\circ} \mathrm{C}$. As one should expect |see Table 3] the increase in temperature considerably accelerated the ferrite dissolution. Zinc oxide slightly prevails over ferric oxide during the first minutes of dissolution at $20^{\circ} \mathrm{G}$. Table 4 concerns the mixtures of nitric and hydrochloric acids and aims to prove that very good conditions of molar solubility are obtained at relatively low concentrations of acid mixture

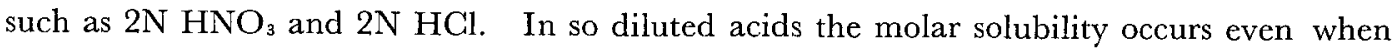
hydrochloric acid accounts for no more than 1/20 of nitric acid, which alone never causes molar solubility as previously mentioned. Due to exceptionally effective solubility in $20: 1$ mixture

Table 2 Solubility of zinc ferrite $\mathrm{ZnFe}_{2} \mathrm{O}_{4}$ versus hydrochloric acid concentration.

\begin{tabular}{|c|c|c|c|c|c|c|}
\hline $\begin{array}{l}\text { Item } \\
\text { No }\end{array}$ & $\begin{array}{c}\text { Acid } \\
\text { concentration, } \\
\text { temperature }\end{array}$ & $\begin{array}{c}\text { Dissol. } \\
\text { time }\end{array}$ & $\begin{array}{c}\mathrm{ZnO} \\
\text { solubility }\end{array}$ & $\begin{array}{c}\mathrm{Fe}_{2} \mathrm{O}_{3} \\
\text { solubility }\end{array}$ & $\underset{\text { solubility }}{\mathrm{ZnO}}$ & $\begin{array}{c}\mathrm{Fe}_{2} \mathrm{O}_{3} \\
\text { solubility }\end{array}$ \\
\hline & normality & $\min$. & $\frac{\mathrm{mg}}{100 \mathrm{ml} \text { of sol. }}$ & $\frac{\mathrm{mg}}{100 \mathrm{ml} \text { of sol. }}$ & $\%$ & $\%$ \\
\hline 1 & 2 & 3 & 4 & 5 & 6 & 7 \\
\hline 1. & $5 \mathrm{~N} \mathrm{HGl}, 40^{\circ} \mathrm{C}$ & $\begin{array}{r}30 \\
60 \\
90 \\
130 \\
200 \\
300 \\
360\end{array}$ & $\begin{array}{r}6.75 \\
10.12 \\
11.81 \\
16.88 \\
27.00 \\
33.76 \\
38.82\end{array}$ & $\begin{array}{r}8.94 \\
13.57 \\
23.18 \\
33.45 \\
53.32 \\
66.57 \\
75.51\end{array}$ & $\begin{array}{r}2.0 \\
3.0 \\
3.5 \\
5.0 \\
8.0 \\
10.0 \\
11.5\end{array}$ & $\begin{array}{r}1.35 \\
2.05 \\
3.50 \\
5.05 \\
8.05 \\
10.05 \\
11.40\end{array}$ \\
\hline 2. & $8 \mathrm{~N} \mathrm{HCl}, 40^{\circ} \mathrm{C}$ & $\begin{array}{r}20 \\
60 \\
100 \\
200 \\
300 \\
350\end{array}$ & $\begin{array}{l}10.12 \\
23.63 \\
35.44 \\
64.14 \\
84.40 \\
91.15\end{array}$ & $\begin{array}{r}19.20 \\
46.36 \\
68.88 \\
126.51 \\
166.26 \\
179.17\end{array}$ & $\begin{array}{r}3.0 \\
7.0 \\
10.5 \\
19.0 \\
25.0 \\
27.0\end{array}$ & $\begin{array}{r}2.90 \\
7.00 \\
10.40 \\
19.10 \\
25.10 \\
27.05\end{array}$ \\
\hline 3. & $10 \mathrm{~N} \mathrm{HCl}, 40^{\circ} \mathrm{C}$ & $\begin{array}{r}30 \\
60 \\
90 \\
120 \\
200 \\
300 \\
350\end{array}$ & $\begin{array}{r}50.64 \\
77.64 \\
101.28 \\
118.16 \\
151.92 \\
175.55 \\
182.30\end{array}$ & $\begin{array}{l}126.18 \\
152.35 \\
198.72 \\
231.84 \\
298.08 \\
342.46 \\
356.37\end{array}$ & $\begin{array}{l}15.0 \\
23.0 \\
30.0 \\
35.0 \\
45.0 \\
52.0 \\
54.0\end{array}$ & $\begin{array}{r}19.05 \\
23.00 \\
30.00 \\
35.00 \\
45.00 \\
51.70 \\
53.80\end{array}$ \\
\hline
\end{tabular}


Table 3 Effect of temperature upon zinc ferrite $\mathrm{ZnFe}_{2} \mathrm{O}_{4}$ solubility in $8 \mathrm{~N} \mathrm{HCl}$.

\begin{tabular}{|c|c|c|c|c|c|c|}
\hline $\begin{array}{l}\text { Item } \\
\text { No }\end{array}$ & $\begin{array}{c}\text { Acid } \\
\text { concentration, } \\
\text { temperature }\end{array}$ & $\begin{array}{c}\text { Dissol. } \\
\text { time }\end{array}$ & $\begin{array}{c}\mathrm{ZnO} \\
\text { solubility }\end{array}$ & $\begin{array}{c}\mathrm{Fe}_{2} \mathrm{O}_{3} \\
\text { solubility }\end{array}$ & $\underset{\text { solubility }}{\mathrm{ZnO}}$ & $\begin{array}{c}\mathrm{Fe}_{2} \mathrm{O}_{3} \\
\text { solubility }\end{array}$ \\
\hline & \multirow{2}{*}{ normality } & \multirow{2}{*}{$\min }$. & $\mathrm{mg}$ & $\mathrm{mg}$ & \multirow{2}{*}{$\%$} & \multirow{2}{*}{96} \\
\hline & & & $100 \mathrm{ml}$ of sol. & $100 \mathrm{ml}$ of sol. & & \\
\hline 1 & 2 & 3 & 4 & 5 & 6 & 7 \\
\hline \multirow{8}{*}{1.} & \multirow{8}{*}{$8 \mathrm{~N} \mathrm{HGl}, 20^{\circ} \mathrm{G}$} & 20 & 3.37 & 6.29 & 1.0 & 0.9 \\
\hline & & 40 & 6.07 & 12.05 & 1.8 & 1.8 \\
\hline & & 60 & 7.42 & 14.57 & 2.2 & 2.2 \\
\hline & & 80 & 10.12 & 20.20 & 3.0 & 3.0 \\
\hline & & 130 & 13.50 & 26.49 & 4.0 & 4.0 \\
\hline & & 190 & 17.55 & 34.44 & 5.2 & 5.2 \\
\hline & & 250 & 25.32 & 49.34 & 7.5 & 7.4 \\
\hline & & 300 & 30.38 & 59.94 & 9.0 & 9.0 \\
\hline \multirow{8}{*}{2.} & \multirow{8}{*}{$8 \mathrm{~N} \mathrm{HCl}, 40^{\circ} \mathrm{C}$} & 20 & 10.12 & 19.87 & 3.0 & 3.0 \\
\hline & & 60 & 23.63 & 46.36 & 7.0 & 7.0 \\
\hline & & 100 & 35.44 & 69.55 & 10.5 & 10.5 \\
\hline & & 150 & 50.64 & 98.03 & 15.0 & 14.8 \\
\hline & & 200 & 64.14 & 127.18 & 19.0 & 19.2 \\
\hline & & 250 & 70.89 & 139.10 & 21.0 & 21.0 \\
\hline & & 300 & 84.40 & 165.60 & 25.0 & 25.0 \\
\hline & & 350 & 91.15 & 177.52 & 27.0 & 26.8 \\
\hline \multirow{8}{*}{3.} & \multirow{8}{*}{$8 \mathrm{~N} \mathrm{HCl}, 60^{\circ} \mathrm{C}$} & 25 & 57.39 & 113.27 & 17.0 & 17.1 \\
\hline & & 40 & 91.15 & 179.17 & 27.0 & 27.0 \\
\hline & & 50 & 111.40 & 219.25 & 33.0 & 33.1 \\
\hline & & 80 & 135.04 & 266.28 & 40.0 & 40.2 \\
\hline & & 130 & 168.80 & 327.88 & 50.0 & 49.5 \\
\hline & & 180 & 189.05 & 372.93 & 56.0 & 56.3 \\
\hline & & 250 & 205.93 & 405.38 & 61.0 & 61.2 \\
\hline & & 300 & 216.06 & 425.92 & 64.0 & 64.2 \\
\hline
\end{tabular}

of $2 \mathrm{~N} \mathrm{NHO}_{3}$ and $2 \mathrm{~N} \mathrm{HCl}$, the effect of temperature upon this process was examined. The results for 20,40 and $60^{\circ} \mathrm{C}$ are set out in Table 5 .

Use was made of the obtained results for searching of the kinetic equation which would reflect best of all the described chemical process. These results allowed to establish the kinetic equation of dissolution and to calculate the dissolution velocity constants $\mathrm{K}$ and $\mathrm{k}$. The so called method of substitution into formula was applied for calculating the kinetic equation of dissolution of the ferrites under examination. This method consists in substitution of experimental data into the expressions for velocity constant, for different equation orders and then in finding this equation which fulfils the requirement of invariability of constant's value.

The zinc ferrite dissolution velocity in acids was studied in connection with specific surface.

If partial solubility of the ferrite in acid is proportional to specific surface then dissolution velocity may be expressed in the form of following general equation:

$$
\frac{\mathrm{dx}}{\mathrm{dt}}=\mathrm{s}_{0} \mathrm{k}|1-\mathrm{x}|^{\mathrm{n}} \quad \mathrm{K}=2 \mathrm{k} \mathrm{s}_{0} \mid \text { for } \mathrm{n}=3 \mid
$$

see page 1 for symbols. 
Table 4 Effect of nitric and hydrochloric acid mixture upon zinc ferrite $\mathrm{ZnFe}_{2} \mathrm{O}_{4}$ solubility.

\begin{tabular}{|c|c|c|c|c|c|c|}
\hline $\begin{array}{l}\text { Item } \\
\text { No }\end{array}$ & $\begin{array}{c}\text { Acid } \\
\text { concentration, } \\
\text { temperature }\end{array}$ & $\begin{array}{c}\text { Dissol. } \\
\text { time }\end{array}$ & $\begin{array}{c}\mathrm{ZnO} \\
\text { solubility }\end{array}$ & $\begin{array}{c}\mathrm{Fe}_{2} \mathrm{O}_{3} \\
\text { solubility }\end{array}$ & $\begin{array}{l}\mathrm{ZnO} \\
\text { solubility }\end{array}$ & $\begin{array}{c}\mathrm{Fe}_{2} \mathrm{O}_{3} \\
\text { solubility }\end{array}$ \\
\hline & normality & $\min$. & $\frac{\mathrm{mg}}{100 \mathrm{ml} \text { of sol. }}$ & $\frac{\mathrm{mg}}{100 \mathrm{ml} \text { of sol. }}$ & $\%$ & 96 \\
\hline 1 & 2 & 3 & 4 & 5 & 6 & 7 \\
\hline 1. & $\begin{array}{l}8 \mathrm{~N} \mathrm{HCl}: 8 \mathrm{~N}^{-\mathrm{HNO}_{3}} \\
=5: 1,40^{\circ} \mathrm{C}\end{array}$ & $\begin{array}{r}20 \\
60 \\
100 \\
190 \\
260 \\
300\end{array}$ & $\begin{array}{r}3.4 \\
10.1 \\
13.5 \\
21.9 \\
25.3 \\
27.0\end{array}$ & $\begin{array}{r}6.3 \\
19.2 \\
26.1 \\
42.4 \\
49.0 \\
54.3\end{array}$ & $\begin{array}{l}1.0 \\
3.0 \\
4.0 \\
6.5 \\
7.5 \\
8.0\end{array}$ & $\begin{array}{l}0.9 \\
2.9 \\
3.9 \\
6.4 \\
7.4 \\
8.2\end{array}$ \\
\hline 2. & $\begin{array}{l}8 \mathrm{~N} \mathrm{HCl}: 8 \mathrm{~N} \mathrm{HNO}_{3} \\
=3: 1,40^{\circ} \mathrm{C}\end{array}$ & $\begin{array}{r}20 \\
60 \\
100 \\
160 \\
260 \\
300\end{array}$ & $\begin{array}{r}6.7 \\
13.5 \\
18.5 \\
23.6 \\
30.4 \\
33.7\end{array}$ & $\begin{array}{l}13.6 \\
25.8 \\
35.7 \\
49.7 \\
59.6 \\
66.2\end{array}$ & $\begin{array}{r}2.0 \\
4.0 \\
5.5 \\
7.0 \\
9.0 \\
10.0\end{array}$ & $\begin{array}{r}2.0 \\
3.9 \\
5.4 \\
7.5 \\
9.0 \\
10.0\end{array}$ \\
\hline 3. & $\begin{array}{l}8 \mathrm{~N} \mathrm{HCl}: 8 \mathrm{~N} \mathrm{HNO}_{3} \\
=1: 1,40^{\circ} \mathrm{C}\end{array}$ & $\begin{array}{r}20 \\
60 \\
100 \\
160 \\
220 \\
300\end{array}$ & $\begin{array}{r}8.4 \\
16.8 \\
25.3 \\
33.7 \\
42.2 \\
50.6\end{array}$ & $\begin{array}{l}16.5 \\
29.8 \\
49.0 \\
66.5 \\
82.8 \\
99.3\end{array}$ & $\begin{array}{r}2.5 \\
5.0 \\
7.5 \\
10.0 \\
12.5 \\
15.0\end{array}$ & $\begin{array}{r}2.5 \\
4.5 \\
7.4 \\
10.0 \\
12.5 \\
15.0\end{array}$ \\
\hline 4. & $\begin{array}{l}2 \mathrm{~N} \mathrm{HNO}_{3}: 2 \mathrm{~N} \mathrm{HCl} \\
=20: 1,40^{\circ} \mathrm{G}\end{array}$ & $\begin{array}{r}80 \\
160 \\
200 \\
300\end{array}$ & $\begin{array}{r}5.7 \\
11.4 \\
13.8 \\
21.6\end{array}$ & $\begin{array}{l}11.26 \\
22.52 \\
27.15 \\
42.40\end{array}$ & $\begin{array}{l}1.7 \\
3.4 \\
4.1 \\
6.4\end{array}$ & $\begin{array}{l}1.7 \\
3.4 \\
4.1 \\
6.4\end{array}$ \\
\hline 5. & $\begin{array}{l}2 \mathrm{~N} \mathrm{HNO}_{3}: 2 \mathrm{~N} \mathrm{HCl} \\
=30: 1,40^{\circ} \mathrm{C}\end{array}$ & $\begin{array}{r}20 \\
60 \\
80 \\
180 \\
240 \\
300\end{array}$ & $\begin{array}{r}5.73 \\
8.40 \\
9.11 \\
13.50 \\
15.19 \\
15.86\end{array}$ & $\begin{array}{r}4.6 \\
9.9 \\
13.2 \\
19.8 \\
24.5 \\
26.5\end{array}$ & $\begin{array}{l}1.7 \\
2.5 \\
2.7 \\
4.0 \\
4.5 \\
4.7\end{array}$ & $\begin{array}{l}0.7 \\
1.5 \\
2.0 \\
3.0 \\
3.7 \\
4.0\end{array}$ \\
\hline
\end{tabular}

Experimental data were substituted into the expressions for dissolution velocity constants received as a result of integrating the following equations:

$$
\begin{aligned}
& \frac{\mathrm{dx}}{\mathrm{dt}}=\mathrm{s}_{0} \mathrm{k}|\mathrm{l}-\mathrm{x}|^{\frac{1}{2}} \quad \mathrm{~K}=\frac{\mathrm{l}}{\mathrm{t}} \mathrm{l}-|1-\mathrm{x}|^{\frac{1}{2}} \quad \mathrm{~K}=\frac{1}{2} \mathrm{ks} \\
& \frac{\mathrm{d} \mathrm{x}}{\mathrm{dt}}=\mathrm{s}_{0} \mathrm{k}|\mathrm{l}-\mathrm{x}|^{\frac{2}{3}} \quad \mathrm{~K}=\frac{\mathrm{l}}{\mathrm{t}} \mathrm{l}-|1-\mathrm{x}|^{\frac{1}{3}} \quad \mathrm{~K}=\frac{\mathrm{l}}{3} \mathrm{ks}_{0} \\
& \frac{\mathrm{dx}}{\mathrm{dt}}=\mathrm{s}_{\mathrm{o}} \mathrm{k}|1-\mathrm{x}|^{1} \quad \mathrm{~K}=\frac{2.303}{\mathrm{t}} \log \frac{1}{1-\mathrm{x}} \\
& \frac{\mathrm{dx}}{\mathrm{dt}}=\mathrm{s}_{0} \mathrm{k}|1-\mathrm{x}|^{2} \quad \mathrm{~K}=\frac{\mathrm{l}}{\mathrm{t}}\left[\frac{1}{1-\mathrm{x}}-1\right] \quad \mathrm{K}=\mathrm{k} \mathrm{s}_{0} \\
& \frac{\mathrm{dx}}{\mathrm{dt}}=\mathrm{s}_{0} \mathrm{k}|1-\mathrm{x}|^{3} \quad \mathrm{~K}=\frac{1}{\mathrm{t}}\left[\frac{1}{\| 1-\left.\mathrm{x}\right|^{2}}-1\right] \quad \mathrm{K}=2 \mathrm{k} \mathrm{s}
\end{aligned}
$$


Table 5 Effect of temperature upon zinc ferrite $\mathrm{ZnFe}_{2} \mathrm{O}_{4}$ solubility.

\begin{tabular}{|c|c|c|c|c|c|c|}
\hline $\begin{array}{l}\text { Item } \\
\text { No }\end{array}$ & $\begin{array}{c}\text { Acid } \\
\text { concentration, } \\
\text { temperature }\end{array}$ & $\begin{array}{c}\text { Dissol. } \\
\text { time }\end{array}$ & $\begin{array}{c}\mathrm{ZnO} \\
\text { solubility }\end{array}$ & $\begin{array}{c}\mathrm{Fe}_{2} \mathrm{O}_{3} \\
\text { solubility }\end{array}$ & $\begin{array}{c}\mathrm{ZnO} \\
\text { solubility }\end{array}$ & $\begin{array}{c}\mathrm{Fe}_{2} \mathrm{O}_{3} \\
\text { solubility }\end{array}$ \\
\hline & normality & $\min$. & $\mathrm{mg}$ & $\mathrm{mg}$ & \multirow{2}{*}{96} & \multirow{2}{*}{$\%$} \\
\hline & normanty & ImIn. & $100 \mathrm{ml}$ of sol. & $100 \mathrm{ml}$ of sol. & & \\
\hline 1 & 2 & 3 & 4 & 5 & 6 & 7 \\
\hline \multirow{4}{*}{1.} & \multirow{4}{*}{$\begin{array}{l}2 \mathrm{~N} \mathrm{HNO}_{3}: 2 \mathrm{~N} \mathrm{HCl} \\
=20: 1,20^{\circ} \mathrm{G}\end{array}$} & 80 & 3.8 & 7.6 & 1.15 & 1.15 \\
\hline & & 160 & 7.4 & 14.5 & 2.20 & 2.20 \\
\hline & & 200 & 9.1 & 17.8 & 2.70 & 2.70 \\
\hline & & 300 & 13.5 & 26.5 & 4.00 & 4.00 \\
\hline \multirow{4}{*}{2.} & \multirow{4}{*}{$\begin{array}{l}2 \mathrm{~N} \mathrm{HNO}_{3}: 2 \mathrm{~N} \mathrm{HGl} \\
=20: 1,40^{\circ} \mathrm{C}\end{array}$} & 80 & 5.7 & 11.2 & 1.70 & 1.70 \\
\hline & & 160 & 11.4 & 22.5 & 3.40 & 3.40 \\
\hline & & 200 & 13.8 & 27.1 & 4.10 & 4.10 \\
\hline & & 300 & 21.6 & 42.4 & 6.40 & 6.40 \\
\hline \multirow{4}{*}{3.} & \multirow{4}{*}{$\begin{array}{l}2 \mathrm{~N} \mathrm{HNO}_{3}: 2 \mathrm{~N} \mathrm{HCl} \\
=20: 1,60^{\circ} \mathrm{G}\end{array}$} & 60 & 10.1 & 19.9 & 3.00 & 3.00 \\
\hline & & 100 & 18.5 & 36.4 & 5.50 & 5.50 \\
\hline & & 220 & 32.0 & 63.0 & 9.50 & 9.50 \\
\hline & & 300 & 35.4 & 82.8 & 12.50 & 12.50 \\
\hline
\end{tabular}

In Table 6 are given exemplary the values of dissolution velocity constants calculated by substitution of experimental data into five above described equations.

As can be seen from these calculations, the following equation is best satisfied by the obtained results:

$$
\frac{\mathrm{dx}}{\mathrm{dt}}=\mathrm{s}_{0} \mathrm{k}|1-\mathrm{x}|^{3} \quad \mathrm{~K}=\frac{1}{\mathrm{t}}\left[\frac{1}{|1-\mathrm{x}|^{2}}-1\right] \quad \mathrm{K}=2 \mathrm{k} \mathrm{s}
$$

Only $\mathrm{ZnO}$ percentage was taken into account in the equations since the percentage of $\mathrm{Fe}_{2} \mathrm{O}_{3}$ dissolution was almost identical in these cases.

To confirm the correctness of the calculated equation

$$
\frac{\mathrm{dx}}{\mathrm{dt}}=\mathrm{k} \mathrm{s}_{0}|1-\mathrm{x}|^{3}
$$

the dependence

$$
\left[\frac{1}{|1-\mathrm{x}|^{2}}-1\right] \text { on } \mathrm{t}
$$

is shown in Fig. 2.

Table 6 Dissolution velocity constants of $\mathrm{ZnFe}_{2} \mathrm{O}_{4}$ ferrite. Dissolution conditions : 2N $\mathrm{HNO}_{3}: 2 \mathrm{~N} \mathrm{HCl}=20: 1,60^{\circ} \mathrm{C}$ at different $\mathrm{n}$

\begin{tabular}{r|c|c|c|c|c|c}
\hline $\mathrm{t} / \mathrm{min}$. & $\begin{array}{c}\mathrm{x} / \% \\
\text { dissol. }\end{array}$ & $\begin{array}{c}\mathrm{K} / \mathrm{min}^{-1} / \mathrm{for} \\
\mathrm{n}=1 / 2\end{array}$ & $\begin{array}{c}\mathrm{K} \\
\text { for } \\
\mathrm{n}=2 / 3\end{array}$ & $\begin{array}{c}\mathrm{K} \\
\text { for } \\
\mathrm{n}=1\end{array}$ & $\begin{array}{c}\mathrm{K} \\
\text { for } \\
\mathrm{n}=2\end{array}$ & $\begin{array}{c}\mathrm{K} \\
\text { for } \\
\mathrm{n}=3\end{array}$ \\
\hline 60 & 0.030 & 0.00287 & 0.00515 & 0.00063 & 0.00051 & 0.0010 \\
100 & 0.055 & 0.00234 & 0.00380 & 0.00056 & 0.00058 & 0.0011 \\
220 & 0.095 & 0.00140 & 0.00207 & 0.00045 & 0.00047 & 0.0010 \\
300 & 0.125 & 0.00117 & 0.00166 & 0.00044 & 0.00047 & 0.0010 \\
\hline
\end{tabular}




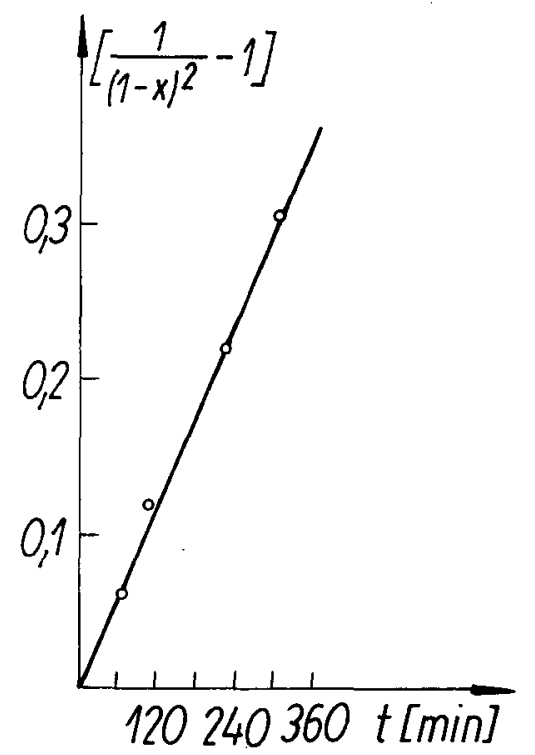

Fig. 2 The dependence of $\left[\frac{1}{(\overline{1}-x)^{2}}-1\right]$ on time.

The calculated dissolution velocity constants $K$ and $k$ are given in Table 7 .

The works at the kinetics of the processes of this type cannot neglect their connections with specific surface and fineness of the sample.

Table 8 comprises the results for zinc ferrite dissolution in hydrochloric acid of molar solubility concentration for two grain fractions. Apparent dissolution velocity constant $\mathrm{K}$, depending on specific surface, was calculated by substitution to formula:

$$
\mathrm{K}=\frac{1}{\mathrm{t}}\left[\frac{1}{|1-\mathrm{x}|^{2}}-1\right]
$$

while velocity constant independent on specific surface was calculated from formula:

Table 7 Values of dissolution velocity constants $\mathrm{K}$ and $\mathrm{k}$ for zinc ferrite $\mathrm{ZnFe}_{2} \mathrm{O}_{4}$.

\begin{tabular}{|c|c|c|c|c|}
\hline $\begin{array}{l}\text { Item } \\
\text { No }\end{array}$ & Acid concentration, temperature & $\mathrm{m}^{2} / \mathrm{g}$ & $\underset{\min ^{-1}}{\mathrm{~K}}$ & $\mathrm{gm}^{-2} \min ^{-1}$ \\
\hline 1 & 2 & 3 & 4 & 5 \\
\hline 1 & $8 \mathrm{~N} \mathrm{HCl}, 20^{\circ} \mathrm{C}$ & 0.4 & 0.000696 & 0.00087 \\
\hline 2 & $8 \mathrm{~N} \mathrm{HCl}, 40^{\circ} \mathrm{C}$ & 0.4 & 0.002528 & 0.00316 \\
\hline 3 & $8 \mathrm{~N}$ HGCl, $60^{\circ} \mathrm{G}$ & 0.4 & 0.021880 & 0.02735 \\
\hline 4 & $5 \mathrm{~N} \mathrm{HCl}, 40^{\circ} \mathrm{C}$ & 0.4 & 0.000953 & 0.00119 \\
\hline 5 & $10 \mathrm{~N} \mathrm{HCl}, 40^{\circ} \mathrm{C}$ & 0.4 & 0.011380 & 0.01422 \\
\hline 6 & $10 \mathrm{~N} \mathrm{HCl}, 80^{\circ} \mathrm{C}$ & 0.4 & 0.245000 & 0.30780 \\
\hline 7 & $2 \mathrm{~N} \mathrm{HNO}_{3}: 2 \mathrm{~N} \mathrm{HCl}=20: 120^{\circ} \mathrm{C}$ & 0.4 & 0.000285 & 0.000356 \\
\hline 8 & $2 \mathrm{~N} \mathrm{HNO}_{3}: 2 \mathrm{~N} \mathrm{HCl}=20: 140^{\circ} \mathrm{C}$ & 0.4 & 0.000440 & 0.000550 \\
\hline 9 & $2 \mathrm{~N} \mathrm{HNO}_{3}: 2 \mathrm{~N} \mathrm{HCl}=20: 160^{\circ} \mathrm{C}$ & 0.4 & 0.001025 & 0.001280 \\
\hline
\end{tabular}


Table 8 Grain size and specific surface effect upon dissolution velocity constants.

\begin{tabular}{|c|c|c|c|c|c|c|c|}
\hline $\begin{array}{c}\text { Item } \\
\text { No }\end{array}$ & $\begin{array}{c}\text { Acid } \\
\text { concentration, } \\
\text { temperature }\end{array}$ & $\begin{array}{l}\text { Grain } \\
\text { size } \\
\mathrm{mm}\end{array}$ & $\begin{array}{c}\mathrm{s}_{0} \\
\mathrm{~m}^{2} / \mathrm{g}\end{array}$ & $\begin{array}{l}\text { Dissol. } \\
\text { time } \\
\text { /min. } /\end{array}$ & $\begin{array}{c}\text { Dissol. } \\
\mathrm{ZnO} \\
\%\end{array}$ & $\begin{array}{c}\mathrm{K} \\
\text { mean value } \\
/ \min ^{-1} /\end{array}$ & \begin{tabular}{|c}
$\mathrm{k}$ \\
mean value \\
$/ \mathrm{g} \cdot \min ^{-1} \mathrm{~m}^{-2} /$ \\
\end{tabular} \\
\hline 1 & 2 & 3 & 4 & 5 & 6 & 7 & 8 \\
\hline 1 & $8.5 \mathrm{~N} \mathrm{HCl}, 40^{\circ} \mathrm{C}$ & $0.033-0.042$ & 0.68 & $\begin{array}{r}60 \\
100 \\
190 \\
300 \\
360\end{array}$ & $\begin{array}{l}16.0 \\
22.0 \\
34.0 \\
44.0 \\
46.0\end{array}$ & 0.006744 & 0.004958 \\
\hline 2 & $8.5 \mathrm{~N} \mathrm{HCl}, 40^{\circ} \mathrm{G}$ & below 0.06 & 0.4 & $\begin{array}{r}60 \\
100 \\
190 \\
300 \\
360\end{array}$ & $\begin{array}{l}10.0 \\
16.0 \\
25.0 \\
32.0 \\
37.0\end{array}$ & 0.004080 & 0.005100 \\
\hline
\end{tabular}

$$
\mathbf{k}=\frac{\mathrm{K}}{2 \mathrm{~s}_{0}}
$$

The results obtained show that specific surface value has considerable effect upon dissolution velocity constant $\mathrm{K}$ whilst dissolution velocity constant $\mathrm{k}$ is in error limits independent on specific surface.

To get more complete picture of the above described zinc ferrite dissolution reactions, Fig. 3 presents k constant dependence on zinc ferrite dissolution temperature in most favourable for this reaction acid mixture of $2 \mathrm{~N} \mathrm{HNO}_{3}: 2 \mathrm{~N} \mathrm{HCl}$ as $20: 1$.

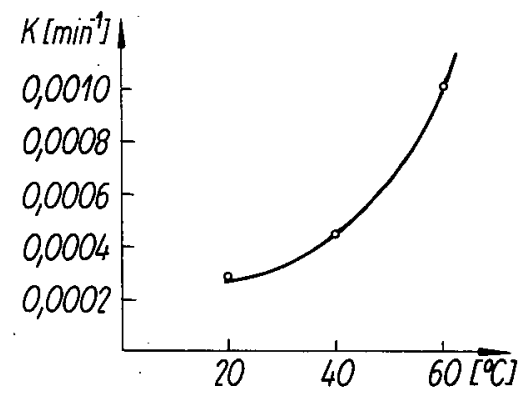

Fig. 3 Constant $|\mathrm{K}|$ dependene on dissolution temperature of $\mathrm{ZnFe}_{2} \mathrm{O}_{4}$ in the mixture of $2 \mathrm{~N} \mathrm{HNO}_{3}: 2 \mathrm{~N} \mathrm{HCl}=20: 1$.

Discussion of error is conducted in order to find the relationship between experimental results and dissolution process correctness. Least squares method was applied to the calculations of accurate values of $\mathrm{K}$ and $\Delta \dot{\mathrm{K}}$ error magnitude. It is common knowledge that in the case of linear relationship between dependent and independent variables

$$
y=a_{1} x+a_{0}
$$

and coefficients $a_{1}$ and $a_{0}$ are calculated from following formulae

$$
\begin{aligned}
& \mathrm{a}_{1}=\frac{\sum \mathrm{x}_{\mathrm{i}} \sum \mathrm{y}_{\mathrm{i}}-\mathbf{n} \sum \mathrm{x}_{\mathrm{i}} \mathrm{y}_{\mathrm{i}}}{\left(\sum \mathrm{x}_{\mathrm{i}}\right)^{2}-\mathrm{n} \sum \mathrm{x}_{\mathrm{i}}{ }^{2}} \\
& \mathrm{a}_{0}=\frac{\sum \mathrm{x}_{\mathrm{i}} \sum \mathrm{x}_{\mathrm{i}} \mathrm{y}_{\mathrm{i}}-\sum \mathrm{y}_{\mathrm{i}} \sum \mathrm{x}_{\mathrm{i}}{ }^{2}}{\left(\sum \mathrm{x}_{\mathrm{i}}\right)^{2}-\mathrm{n} \sum \mathrm{x}_{\mathrm{i}}{ }^{2}}
\end{aligned}
$$


where

$\mathrm{x}_{1}$-measured values of independent variable

$\mathrm{y}_{1}$-measured values of dependent variable

n-number of measurements.

Summation takes place against all measurements taken into consideration. Sometimes, to estimate the degree of adaptation of measuring points to straight line the so called corelation factor is calculated:

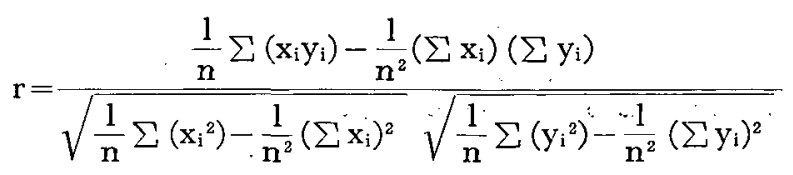

The errors of $\mathrm{a}_{1}$ and $\mathrm{a}_{0}$ are calculated according the formulae:

$$
\begin{aligned}
& \Delta \mathrm{a}_{1}= \pm \sqrt{\frac{\sum\left(\mathrm{y}_{\mathrm{i}}-\mathrm{Y}_{\mathrm{i}}\right)^{2}}{(\mathrm{n}-2)\left(\sum \mathrm{x}_{\mathrm{i}}-\overline{\mathrm{x}} \sum \mathrm{x}_{\mathrm{i}}\right)}} \\
& \Delta \mathrm{a}_{0}= \pm \sqrt{\frac{\sum\left(\mathrm{y}_{\mathrm{i}}-\mathrm{Y}_{\mathrm{i}}\right)^{2}}{\mathrm{n}-2}} \cdot \sqrt{\frac{\sum \mathrm{x}_{\mathrm{i}}{ }^{2}}{\mathrm{n} \sum \mathrm{x}_{\mathrm{i}}{ }^{2}-\left(\sum \mathrm{x}_{\mathrm{i}}\right)^{2}}}
\end{aligned}
$$

where

$\mathrm{Y}_{\mathrm{i}}$-dependent variable value corresponding to independent variable $\mathrm{x}_{\mathbf{i}}$ given by:

$$
y=a_{1} x+a_{0}
$$

In our case $x_{1}$ means time $t$, while

$$
y=\frac{1}{|1-x|^{2}}-1
$$

$a_{1}$ is dissolution velocity constant and theoretically $a_{0}$ should be equal to $O$.

Table 9 contains calculation results concerning the measurements of zinc ferrite dissolution process in $2 \mathrm{~N} \mathrm{HNO}_{3}: 2 \mathrm{~N} \mathrm{HCl}=20: 1$ at $60^{\circ} \mathrm{C}$.

Table 9

\begin{tabular}{c|c|c|c|c|c|c}
\hline $\begin{array}{c}\text { Item } \\
\text { No }\end{array}$ & \multicolumn{1}{c|}{$\mathrm{x}_{\mathrm{i}}$} & $\mathrm{y}_{\mathrm{i}}$ & \multicolumn{1}{c|}{$\mathrm{x}_{\mathrm{i}} \mathrm{y}_{\mathrm{i}}$} & \multicolumn{1}{c|}{$\mathrm{x}_{\mathrm{i}}{ }^{2}$} & $\mathrm{y}_{\mathrm{i}}{ }^{2}$ & $\mathrm{Y}_{\mathrm{i}}$ \\
\hline 1. & 60 & 0.0628 & 3.768 & 3600 & 0.003943 & 0.06999 \\
2. & 100 & 0.1198 & 11.980 & 10000 & 0.014350 & 0.10900 \\
3. & 220 & 0.2210 & 48.620 & 48400 & 0.048840 & 0.22625 \\
4. & 300 & 0.3061 & 91.830 & 90000 & 0.093690 & 0.30438 \\
\hline sum $\Sigma$ & 680 & 0.7097 & 156.198 & 152000 & 0.160823 & \\
\hline
\end{tabular}

Using these data one obtain:

$$
a_{1}=K=0.0009766 \pm 0.0000293
$$

Hence relative error of dissolution velocity constant is $\pm 3 \%$.

It follows that zinc ferrite dissolution process in acids under examination is much better 1971年 7 月 
described by kinetic equation with exponent 3 than with exponent $2 / 3$ given in the Japanese work. But it is absolutely necessary to emphasize that experimental conditions and kind of acid are basically different from those applied in japanese works. This seems to be principal reason of divergences in mathematical treatment of results.

\section{References}

1) K. Nii and Y. Hisamatsu : J. Min. Met. Inst. Japan, 80 (1964) 95

2) K. Nii and Y. Hisamatsu : J. Min. Met. Inst. Japan, 80 (1964) 362

3) K. Nii and Y. Hisamatsu: Trans. of NRIM 8 (1966) 183

4) W. Wolski, U. Politanska and K. Kasprzak: Przemysf Chemiczny 44 (1965) 481

5) W. Wolski and U. Politanska: Monatshefte für Chemie 99 (1968) 733

新入 正会 員

（昭和 46 年 5 月 6 日現在）

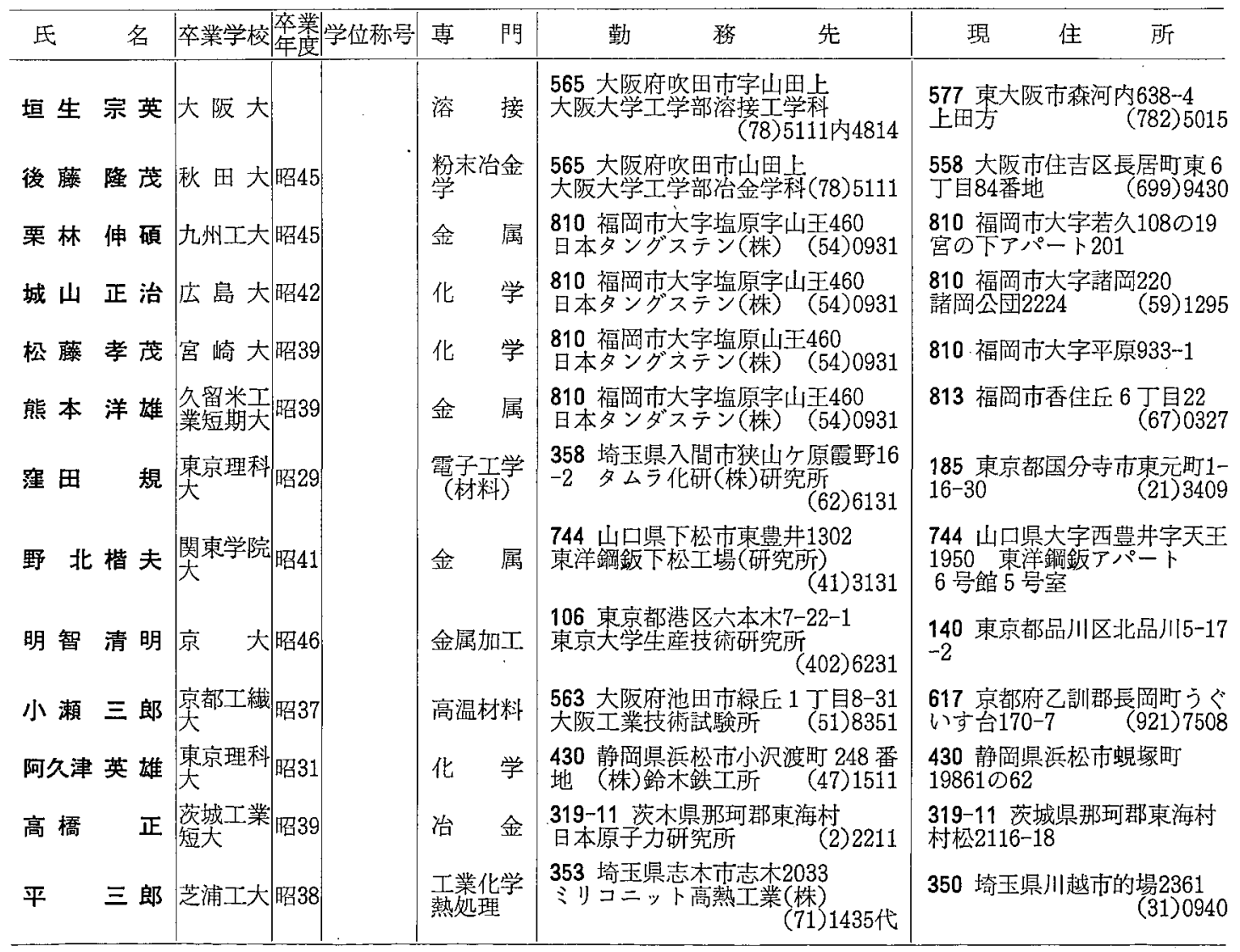

